\title{
Tadpole Conformation of Gradient Polymer Brushes
}

\author{
Samuel J . Lord, ' Sergei S. Sheiko, ${ }^{* \dagger}$ Isaac LaRue, ${ }^{\dagger}$ Hyung-II Lee, ${ }^{\ddagger}$ and \\ Krzysztof Matyjaszewski*,*
}

Department of Chemistry, University of North Carolina at Chape Hill, North Carolina 27599-3290, and Department of Chemistry, Carnegie Mellon University, 4400 Fifth Avenue,

Pittsburgh, Pennsylvania 15213

Received December 23, 2003; Revised Manuscript Received March 23, 2004

\begin{abstract}
Cylindrical molecular brushes, each with a gradient of grafting density along the backbone, demonstrated a transition from rodlike to tadpole conformations. Using atomic force microscopy (AFM), a coexistence of two conformational phases was observed within individual molecules adsorbed on a mica substrate. These observations were made by compressing monolayers on the surface of water and then transferring a sample of this monolayer to a mica substrate for AFM studies. U pon compression, the rod-globule transition occurs at the end where the brush is densely grafted, leaving a molecule with a globular "head" and an extended "tail"-a so-called tadpole conformation. This research demonstrates the asymmetric changes in the molecular conformation, which is one of the prerequisites for directed motion.
\end{abstract}

\section{Introduction}

Cylindrical polymer brushes, also known as molecular bottle brushes, are made by attaching polymer chains al ong a flexible polymer backbone. ${ }^{1-6}$ In solution, these molecules adopt a wormlike conformation stabilized by steric repulsion of densely grafted side chains. ${ }^{7-10}$ When adsorbed, the planar surface breaks the symmetry of the brushes, thus allowing more conformations that depend on the fraction of adsorbed side chains $\varphi_{\mathrm{a}}{ }^{11}$ Previous studies have reported that cylindrical brushes adsorbed on surfaces can undergo a first-order phase transition from a rodlike conformation to a more compact globular conformation when compressed. ${ }^{11,12}$ The transition results from competition of the energetically favorable interaction of side chains with the substrate and entropically unfavorable extension of adsorbed side chains. Because of steric repulsion between the adsorbed side chains, a brush with a high $\varphi_{a}$ adopts a rodlike conformation. Conversely, a brush with a relatively small $\varphi_{a}$ prefers a globular conformation favored by the aggregation of desorbed side chains.

Along with the side-chain length, the grafting density of side chains is another parameter governing this transition;11,12 thus, there is an interest in characterizing this relation. According to theoretical predictions, denser brushes should readily show the rod-globule transition, while loose brushes (those with a low grafting density) preserve their extended conformation. Although conformations of individual brush molecules of differing side-chain density have been reported, ${ }^{11}$ no study has investigated the effect of grafting density on phase transitions or reported phase transitions occurring in only part of a brush molecule. Because these current brushes exhibit a gradient in side-chain density, it is possible to induce a transformation of the denser end of a molecule while the less densely grafted end remains extended (Figure 1). Such coexistence allows examining molecules that exhibit two conformations simulta-

† University of North Carolina at Chapel Hill.

‡ Carnegie Mellon University.

* Corresponding author. E-mail: sergei@email.unc.edu
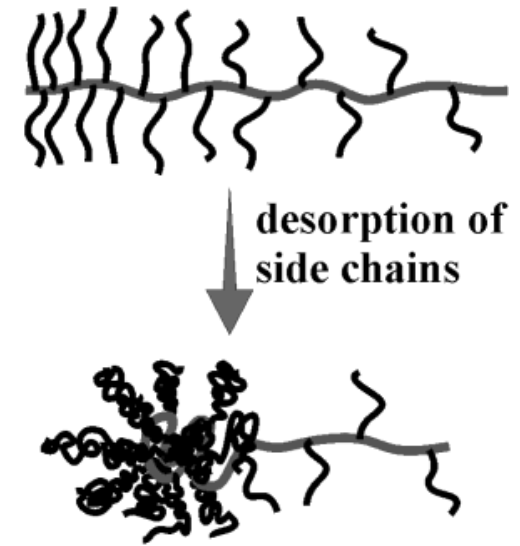

Figure 1. When cylindrical brushes possessing a gradient grafting density al ong the backbone are adsorbed on a surface, one can observe a transition from a rodlike to a tadpole conformation upon partial desorption of side chains. The end with higher grafting density, and thus with a greater extension of the side chains, is predicted to collapse more readily than the loose end.

neously under the same conditions. This study investigates molecules with an asymmetric conformation that may eventually demonstrate directed motion similar to other systems with broken symmetry. ${ }^{13-16}$

\section{Experimental Section}

The gradient brushes used in this experiment are made from spontaneous gradient backbones prepared by copolymerization of methyl methacrylate (MMA) and 2-(trimethyl silyl oxy)ethyl acrylate (HEA-TMS). This contrasts with the previous works dealing with the forced gradient. ${ }^{4}$ In the spontaneous system, the instantaneous composition of the backbone is determined by the initial monomer feed ratios and the monomer reactivity ratios. ${ }^{17-19}$ Because of the higher reactivity ratio of MMA as compared to HEA-TMS, MMA is predominantly incorporated into the backbone in the beginning of polymerization. After most of the MMA is polymerized, HEA-TMS is more favorably incorporated, leading to a spontaneous gradient backbone.

Synthesis of p(MMA-grad-HEA-TMS) Copolymer. In a $10 \mathrm{~mL}$ round-bottom flask, ethyl 2-bromoisobutyrate $(7.34 \mu \mathrm{L}$, $0.05 \mathrm{mmol}$ ) and anisole (1.8 $\mathrm{mL}, 50 \mathrm{vol} \%)$ were combined, and the solution was bubbled for $10 \mathrm{~min}$ at $0{ }^{\circ} \mathrm{C}$. In a $25 \mathrm{~mL}$ 
Schlenk flask, PMDETA (10.4 $\mu \mathrm{L}, 0.05 \mathrm{mmol})$, MMA (2.25 g, $22.5 \mathrm{mmol})$, and HEA-TMS (1.4 g, $7.5 \mathrm{mmol}$ ) were added, and the resulting solution was degassed by three freeze-pumpthaw cycles. $\mathrm{CuBr}(0.007 \mathrm{~g}, 0.05 \mathrm{mmol})$ was added; after stirring for $10 \mathrm{~min}$, the Schlenk flask was placed in a thermostated oil bath at $85^{\circ} \mathrm{C}$. After $3 \mathrm{~min}$, the initiator solution was transferred into the Schlenk flask, and an initial sample was taken. During polymerization, samples were removed to determine molecular weight by gel permeation chromatography (GPC) and conversion by gas chromatography (GC). The reaction mixture was stirred for $7 \mathrm{~h}$, exposed to air, diluted in $\mathrm{CH}_{3} \mathrm{Cl}$, and filtered through a neutral alumina column to remove the copper catalyst. Next, the solvent was removed, and the polymer was dried under vacuum to a constant weight.

Synthesis of Gradient Brushes. The synthesis of p(M MAgrad-HEA-TMS) provided a precursor polymer, which was transformed by cleavage of the TMS protective groups and esterification with 2-bromopropionyl bromide to a p(MMAgrad-BPEA) multifunctional macroinitiator with reactive 2-(2bromopropionyl oxy)ethyl acrylate (BPEA) groups. A series of gradient poly(n-butyl acrylate) (pBA) brushes with different average grafting densities and different lengths of the side chains were prepared by the so-called "grafting from" approach described in the current literature. ${ }^{4}$ Atom transfer radical polymerization (ATRP) ${ }^{20,21}$ was used to grow pBA side chains from the macroinitiator.

Characterization of Polymers. Average molecular weights and molecular weight distributions were measured using GPC equipped with Waters microstyragel columns (pore size $10^{5}$, $10^{4}$, and $10^{3} \AA$ ) and three detection systems: differential refractometry (Waters model 410), multiangle laser light scattering (MALLS) (Wyatt, DAWN EOS), and differential viscometry (WGE Dr. Bures, $\eta$-1001). The gradient in grafting density was characterized using GC by measuring the instantaneous fraction of graftable monomers while synthesizing the macroinitiator.

Sample Preparation. Langmuir-Blodgett (LB) films were prepared using a KSV 5000 instrument and a trough filled with double-distilled water (Milli-Q). F or each experiment, a monolayer film of brushes was compressed at a rate of $10 \mathrm{~mm} /$ min, and then the film was transferred onto a mica substrate at a transfer speed of $1.0 \mathrm{~mm} / \mathrm{min}$. Dense monolayers of each brush were transferred at several different degrees of compression for atomic force microscopy (AFM) measurements. To prepare monolayers in which molecules do not aggregate or contact each other, brushes were mixed with linear pBA $\left(M_{n}\right.$ $=2.1 \times 10^{4} \mathrm{~g} / \mathrm{mol}, M_{w} / M_{n}=1.3$ ) at a $10 / 90 \%$ mass ratio. This provided LB monolayers of single brush molecules embedded in a pBA matrix.

Spin-casting was used as an alternative technique for single molecule studies. To prepare sparse monolayers for AFM analysis, a Laurell Technologies instrument was used to spincast one drop of dilute brush solution (approximately $10^{-2} \mathrm{mg} /$ $\mathrm{mL}$ ) onto a mica substrate at approximately $3000 \mathrm{rpm}$.

Atomic Force Microscopy. AFM images were collected using a Multimode III a atomic force microscope (Veeco M etrology Group). The microscope was operated in tapping mode at ambient conditions $\left(\mathrm{T}=21^{\circ} \mathrm{C}, \mathrm{RH}=45 \%\right)$, using Si cantilevers (Mikromasch) with a resonance frequency of approximately $160 \mathrm{kHz}$ and a tip radius less than $10 \mathrm{~nm}$. To avoid damage or deformation to the samples, the measurements were performed under light tapping conditions using a 0.9 set point.

\section{Results and Discussion}

Molecular Characterization: Weight, Gradient, and Size. Cylindrical brushes with two different gradients of grafting densities were prepared using the grafting-from approach schematically shown in Figure 2. Table 1 displays molecular characteristics of brushes IA, IIA, and IIIB as well as the linear macroinitiators for each. Brushes IA and IIA were prepared from the same macroinitiator $A$ : a linear backbone with an

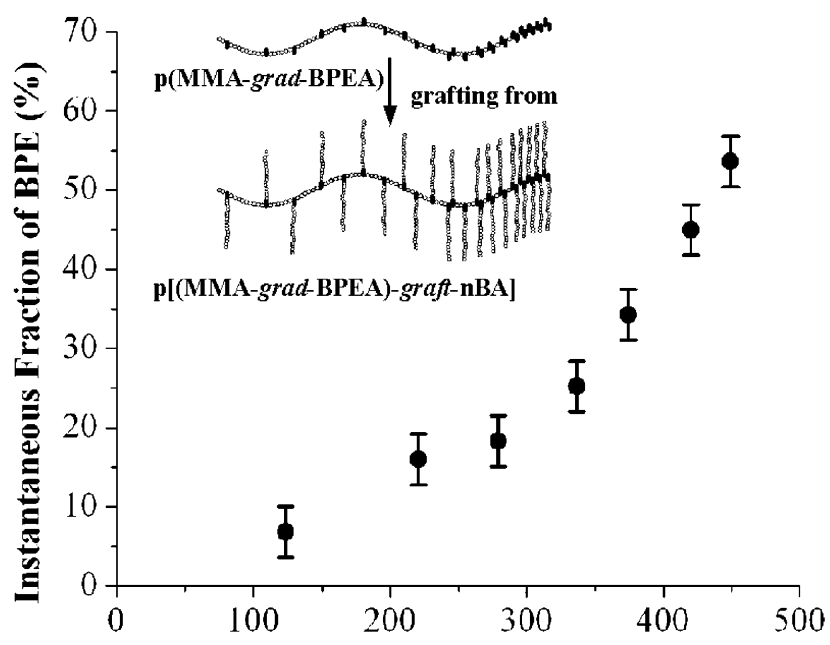

Degree of Polymerization Along Backbone

Figure 2. Gradient brushes were prepared by the grafting from approach, i.e., by polymerization of nBA from a p(MMAgrad-BPEA) macroinitiator possessing a certain gradient of graftable BPEA units al ong the backbone (inset). Instantaneous fraction of graftable BPEA units along the macroinitiator B was calculated from the monomer conversion using the equation $\% \mathrm{IC}=\Delta_{\mathrm{BPE}} /\left(\Delta_{\mathrm{BPE}}+3 \Delta_{\mathrm{MMA}}\right)$, where $\Delta$ is the change in the percent conversion of monomers since the previous measurement. The initial feed ratio of MMA to BPEA precursor was 3:1, so $\Delta_{\mathrm{MMA}}$ is tripled in the denominator.

Table 1. Molecular Weight Characterization of Macroinitiators and Gradient Brushes by MALLS-GPC

\begin{tabular}{cccccr}
\hline sample $^{a}$ & BPEA:MMA $^{b}$ & $\mathrm{M}_{n}{ }^{\mathrm{c}}$ & PDI $^{\mathrm{d}}$ & $\mathrm{Ne}$ & $\mathrm{n}^{\mathrm{f}}$ \\
\hline A & $1: 1$ & $7.1 \times 10^{4}$ & 1.2 & 405 & 0 \\
B & $1: 3$ & $6.7 \times 10^{4}$ & 1.3 & 486 & 0 \\
IA & $1: 1$ & $1.2 \times 10^{6}$ & 1.3 & 405 & 43 \\
IIA & $1: 1$ & $2.0 \times 10^{6}$ & 1.5 & 405 & 74 \\
IIIB & $1: 3$ & $5.7 \times 10^{5}$ & 1.3 & 486 & 32
\end{tabular}

a $A$ and $B$ are two macroinitiators that were used for the synthesis of brush molecules IA, IIA, and IIIB. Polymers IA and IIA were prepared from macroinitiator $A$, while polymer IIIB was prepared from $B .{ }^{b}$ Average molar ratio of methyl methacrylate (MMA) and 2-(2-bromopropionyloxy)ethyl acrylate (BPEA) monomeric units in the gradient macroinitiator. ' Number-average molecular weight measured at a relative standard deviation (RSD) of $5 \%$. d Polydispersity index PDI $=M_{w} / M_{n}$. e Number-average degree of polymerization of the backbone, i.e., macroinitiator, was calculated as $\mathrm{N}=\mathrm{M}_{\mathrm{n}} /\left[(1-\chi) \mathrm{M}_{\mathrm{MMA}}+\chi \mathrm{M}_{\mathrm{BPE}}\right]$, where $\mathrm{M}_{\mathrm{MMA}}=100$ and $M_{\text {BPEA }}=251$ are molecular weights of MMA and BPEA monomeric units, respectively, and $\chi$ is the molar fraction of grafted BPEA units in the backbone, e.g. $\chi=0.25$ for the $1: 3$ molar ratio. The calculation results in RSD $=10 \% .{ }^{\mathrm{f}}$ Number-average degree of polymerization of the $\mathrm{pBA}$ side chains was calculated as $n=\left(M_{n, b r u s h}-M_{n}\right.$,macroinitiator $) / \chi N M_{B A}$, where $M_{B A}=128$ is molecular weight of $n$-butyl acrylate (BA).

approximate 1:1 ratio of graftable BPEA and nonfuncti onalized MMA monomers. From MALLS-GPC analysis, number-average degrees of polymerization $n=43$ and $n=74$ were obtained for the side chains of polymers IA and IIA, respectively. Conversely, brush IIIB has an approximate 1:3 ratio of BPEA to MMA monomers, thus decreasing the density of grafted side chains and possibly increasing the gradient. The number-average degree of polymerization of the side chains for brush IIIB is less than that for the other two brushes ( $n=$ 32).

The grafting density gradient was characterized during synthesis of the macroinitiators. Several times during the reactions, the percent conversion of comonomers was measured using GC. From these measure- 

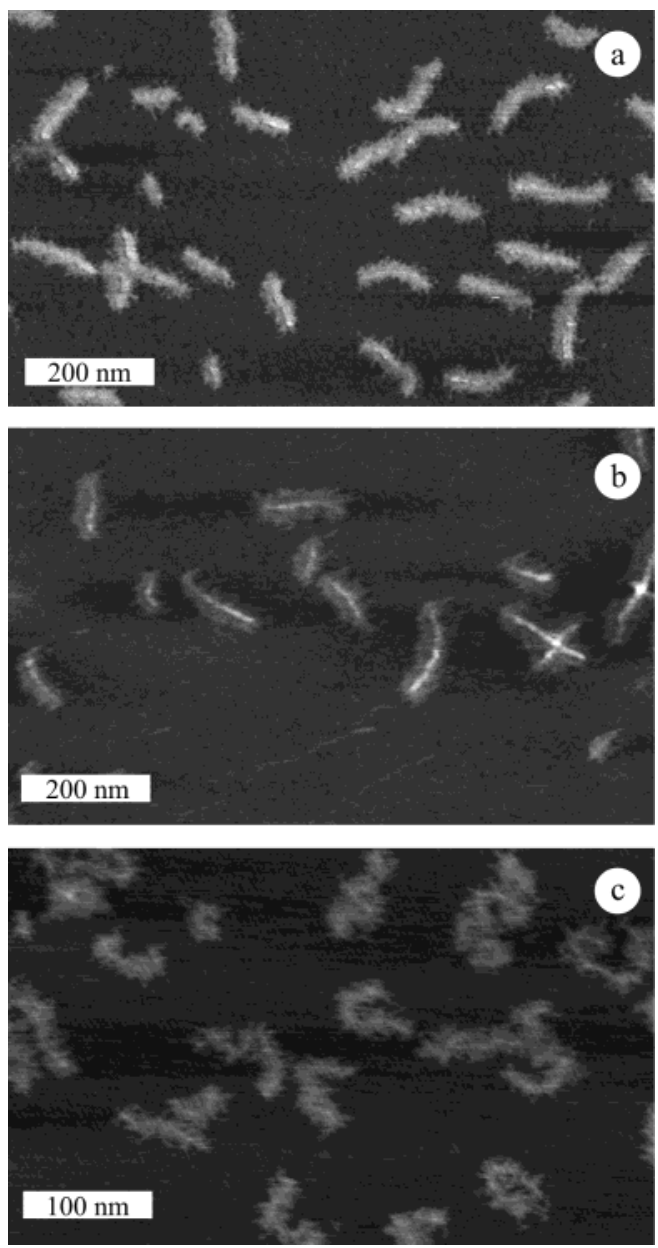

Figure 3. Height micrographs of single brush molecules adsorbed on mica were measured by tapping-mode AFM. The micrographs correspond to different brush molecules. (a) Polymer IA was synthesized from macroi nitiator A, which has a 1:1 grafting ratio (graftable units to nonreactive units). (b) Polymer II A was al so synthesized from macroinitiator A, but its side chains are nearly twice as long as those in polymer IA. Note the larger corona and more obvious gradient in the light backbone thread for polymer IIA. (c) Polymer IIIB was synthesized from macroinitiator $B$, which has a 1:3 grafting ratio and has shorter side chains than those on polymers IA and IIA. The lower grafting density of polymer IIIB allows the brushes to be less extended and have greater curvature.

ments, the instantaneous fraction of the graftable BPEA units in the backbone was calculated. The gradient in this instantaneous fraction along the length of the backbone should reflect the gradient in grafting density of the final bottle brush. Figure 2 displays the instantaneous fraction of macroinitiator $B$ with respect to degree of polymerization along the chain. The plot shows a clear gradient in fraction of the B macroinitiator, which is only approximately 5\% BPEA at one end and increases to nearly $55 \%$ at the other end.

Molecular size was also studied by AFM provided that individual molecules are clearly resolved. Figure 3 shows height images of single molecules of brushes I A, IIB, and IIIB adsorbed from a dilute solution on mica via spin-casting. All the molecules clearly exhibit a hairy corona of the side chains around the backbone. In some molecules, a white thread highlights the backbone of the bottle brushes. This topographic contrast between the backbone and the corona results from desorbed side chains that congregate around the backbone while the adsorbed side chains lie flat on the surface. Since

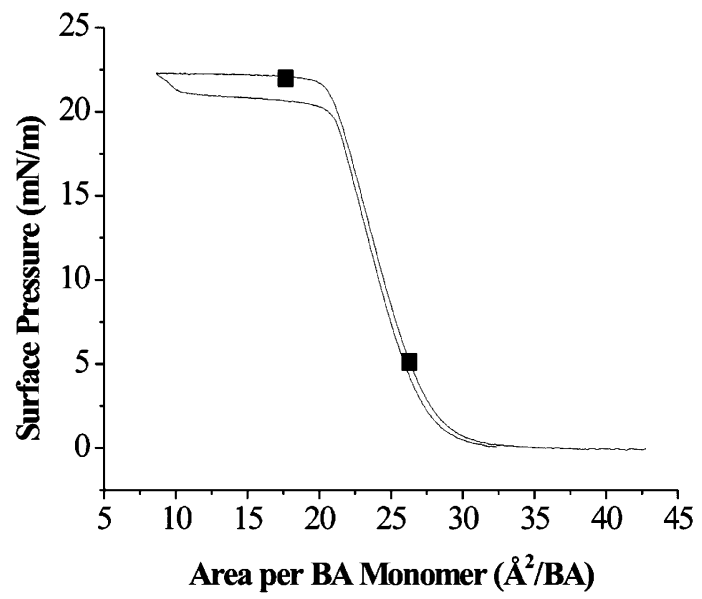

Figure 4. Surface pressure-molecular area isotherm measured for a monolayer of sample la on the surface of water. The molecular area was calculated as film area per n-butyl acrylate (BA) monomeric unit. Films were transferred to mica substrates at different pressures (marked by squares) for AFM measurements.

desorption readily occurs at the denser end of the brush, one should expect to observe a thicker thread at one of the ends of the visualized molecules. The thickness gradient is clearly visible for polymer IIA with longer side chains (Figure $3 b$ ). Here, one can see how the thickness of the thread increases along the brush backbone. One can also note that molecules I A and II A are more extended and thus exhibit less curvature compared to molecules IIIB. We ascribe this effect to the higher grafting density of polymers I A and II A: at higher grafting densities, the backbone is more extended due to stronger repulsion between the adsorbed side chains.

Molecular visualization was also achieved for dense monolayers prepared by the Langmuir-Blodgett technique. The studied polymers showed very similar surface pressure vs molecular area isotherms. Figure 4 shows the isotherm measured for a dense monolayer film of sample la. To visualize individual molecules, the film was transferred at a low pressure of $5 \mathrm{mN} / \mathrm{m}$. Figures $5 \mathrm{a}$ and $6 \mathrm{a}$ show the molecular structure of LB films prepared from brush molecules as such and from a mixture of brush molecules with linear pBA. In Figure $5 a$, one can see densely packed wormlike molecules, while in the sparse monolayer in Figure 6a, the molecules are separated by a monolayer of linear pBA. Other images in Figures 5 and 6 show conformational changes caused by compression, which will be discussed in the second section of the paper.

Molecular visualization allowed size measurements for a large ensemble of individual molecules. ${ }^{22-27}$ Table 2 summarizes the data collected for both sparse and dense monolayers. The polydispersity values range from 1.1 to 1.2, which is consistent with the polydispersity of other macroinitiators prepared by the ATRP technique. ${ }^{3,4,12}$ To eval uate axial contraction, we cal culated the length per monomeric unit $\mathrm{I}_{\mathrm{m}}=\mathrm{L}_{\mathrm{n}} / \mathrm{N}$ of the main chain, where $L_{n}$ is the number-average contour length of the backbone and $\mathrm{N}$ is the number-average degree of polymerization of the backbone. These values can be no more than $2.5 \AA$-the projected length of a carbon chain in the all-trans (zigzag) conformation-because the main chain cannot be any longer than its fully extended length. The brush with the greater grafting density and the longest side chains (IIA) has the highest $I_{m}$ value 

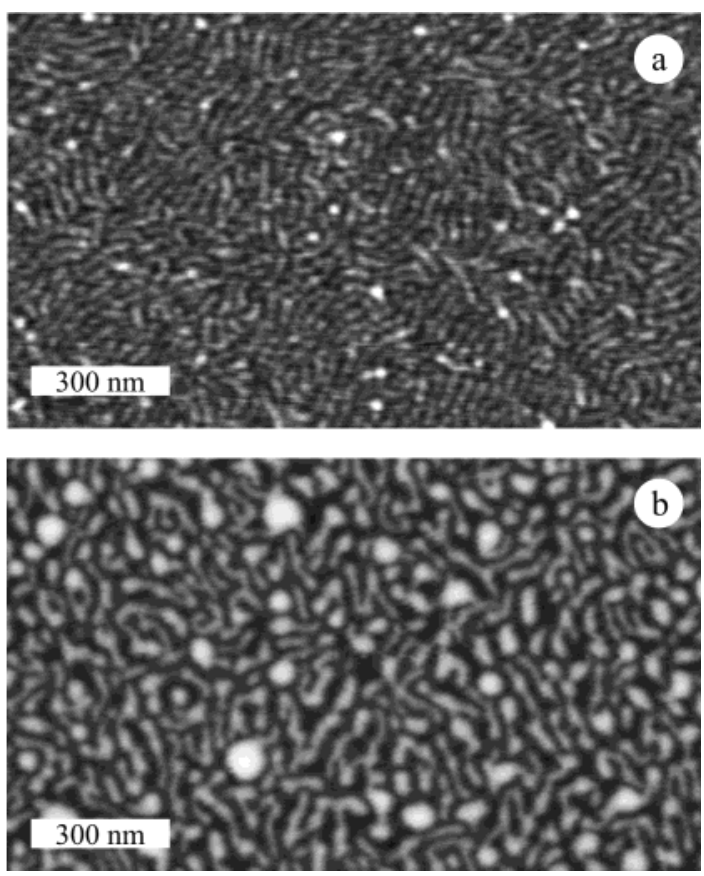

Figure 5. AFM micrographs show molecular resolution of dense monolayers of gradient brushes IA prepared by the Langmuir-Blodgett technique. The monolayer were transferred onto mica surface at different molecular areas $A$ and pressures $\pi$. (a) At $A=26 \AA^{2} / B A$ and $\pi=5 \mathrm{mN} / \mathrm{m}$, the dense monolayer consists of rodlike brushes. (b) At a higher degree of compression $\left(A=17 \AA^{2} / \mathrm{BA}, \pi=22 \mathrm{mN} / \mathrm{m}\right)$, many molecules take on the tadpole conformation. Samples of brushes IIA and IIIB exhibited similar conformation change.
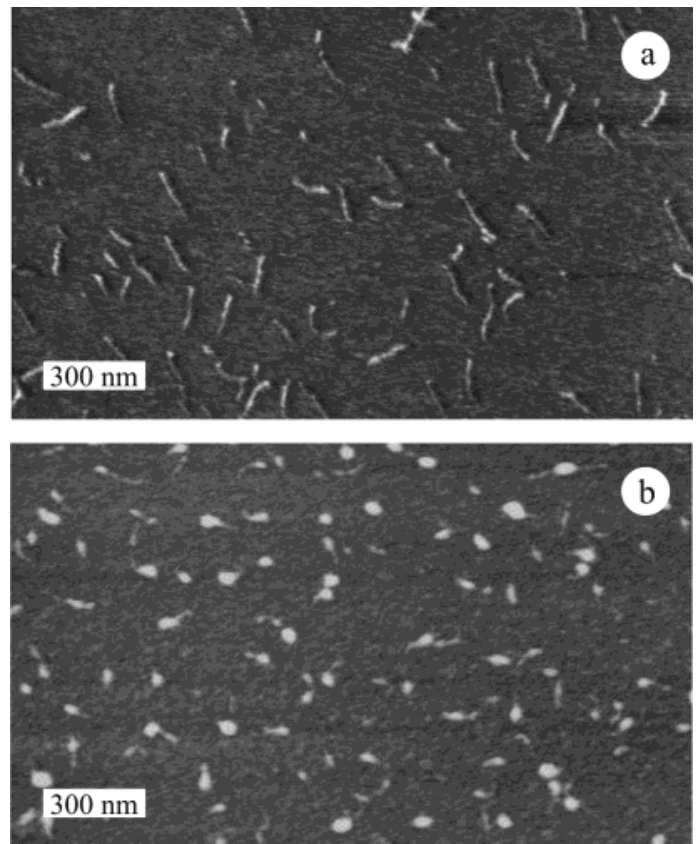

Figure 6. AFM micrographs show single molecules of gradient brushes IIA embedded in a matrix of linear pBA in order to better observe the molecular transition, which was obscured by the neighbors in dense monolayers (Figure 5). The micrographs correspond to different degrees of compression. (a) At $\mathrm{A}=26 \AA^{2} / \mathrm{BA}$ and $\pi=5 \mathrm{mN} / \mathrm{m}$, the rodlike brushes reveal a gradient in width and height more clearly than in a dense monolayer. (b) At $\mathrm{A}=17 \AA^{2} / \mathrm{BA}$ and $\pi=22 \mathrm{mN} / \mathrm{m}$, the brushes exhibit their tadpole shape clearly against the background of linear pBA.

and thus is extended to the greatest degree. This extension causes brush II A to exhibit a larger number-
Table 2. Dimensions of Gradient Brushes on a Surface

\begin{tabular}{ccccc}
\hline sample & $\mathrm{L}_{n}{ }^{\mathrm{a}}(\mathrm{nm})$ & $\mathrm{PDI}^{\mathrm{b}}$ & $\mathrm{Im}^{\mathrm{c}}(\mathrm{nm})$ & $\mathrm{D}^{\mathrm{d}}(\mathrm{nm})$ \\
\hline IA & $70 \pm 5$ & 1.12 & $0.17 \pm 0.02$ & $24 \pm 1$ \\
IIA & $81 \pm 5$ & 1.14 & $0.20 \pm 0.02$ & $32 \pm 1$ \\
IIIB & $71 \pm 5$ & 1.20 & $0.15 \pm 0.02$ & $15 \pm 1$
\end{tabular}

a Number-average length of the backbone of an ensemble of approximately 100-200 single mol ecules (corrected for side chains protruding at ends by subtracting the width $D$ from the length of the entire molecule). ${ }^{b}$ Polydispersity index PDI $=L_{w} / L_{n} .{ }^{c}$ Length per monomeric unit of the backbone $I_{m}=L_{n} / N$. ${ }^{d}$ Average distance between molecules in a dense monolayer.

average length than IA, even though they share the same macroinitiator. Although the length differences are within the experimental error, higher extension of molecules IIA is to be expected due to greater steric repulsion of the longer and denser side chains.

Table 2 also presents the approximate width of individual molecules within a dense monolayer (Figure 5). The width of the brushes in the substrate plane was measured to be about 24 and $32 \mathrm{~nm}$ for polymers IA and IIA, respectively. This difference is consistent with the longer side chains of polymer IIA, since the sidechain length controls the distance between the neighboring brush molecules.

Conformational Transition. A previous publication reported a transition of individual brushes from the rodlike phase to a globular conformation upon compression on an LB trough. ${ }^{12}$ When monolayers of brushes were compressed, side chains desorbed and the molecules transformed into globules. It was shown that this change was a first-order phase transition, where the fraction of adsorbed side chains depends on the length of the side chains and the grafting density. Since the current brushes exhibit a gradient in side-chain grafting density, we anticipated a rod-globule transition to occur in the denser part of the brush molecules, which experiences a larger entropic penalty due to extension of adsorbed side chains. To verify this hypothesis, we studied conformational changes caused by lateral compression of brush molecules on the surface of water.

In Figure 4, the onset of pressure was observed at 30 $\AA^{2}$ (a typical value for linear pBA), which was followed by a linear increase of pressure in the range from 30 to $21 \AA^{2}$, where the pressure leveled off to give a plateau at $22.5 \mathrm{mN} / \mathrm{m}$. The isotherm also depicts the molecular areas at which transfers were carried out. At different pressures, the molecules had noticeably different conformations. Figure 5 shows molecular structure of dense monolayers transferred on a mica substrate at two degrees of compression. Molecules at both pressures reveal heterogeneous structure in which the width of the molecules varies along the backbone. In Figure 5b, one can see that some molecules possess both a globule head and a tail. However, in these dense monolayers, it is difficult to identify the individual molecules, especially their ends.

To more clearly observethese conformational changes, brush molecules of sample IIA were isolated by mixing them with linear pBA. Figure 6 shows AFM images obtained upon compression of the monolayer prepared from this mixture. The brush molecules are separated by a homologous film of linear pBA to screen attractive forces between adsorbed brush molecules. Thus, not only does the conformation change become more noticeablethe tadpole structure is unmistakable-but also the gradient in the rodlike molecules is more evident: in 

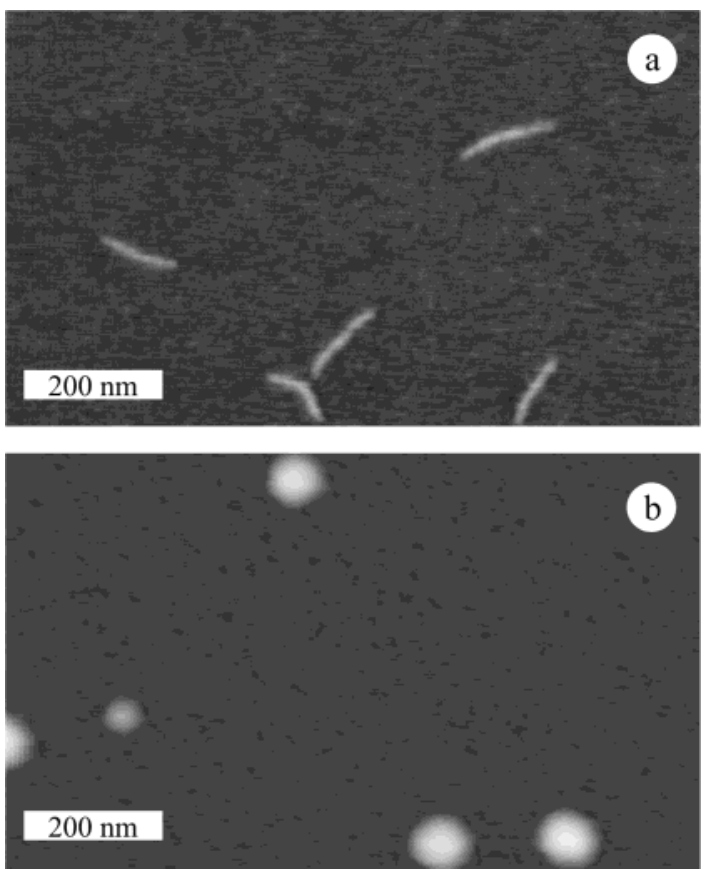

Figure 7. AFM height micrographs demonstrate the transition from the rodlike to a symmetrical gl obular conformation of homogeneous bottle-brush molecules (without gradient of grafting density) embedded in a pBA matrix. (a) Before compression, each brush exhibits the rodlike conformation. (b) The entire molecule collapses into a symmetrical gl obule upon compression.

many molecules, one end is clearly thinner than the other.

The observed transition can be compared with those of regular bottle brush molecules (i.e., homogeneous brushes without gradient of grafting density). A sample of homogeneous brushes was prepared in the same way, using the LB technique applied to a mixture of brush molecules ( $\mathrm{N}=570, \mathrm{n}=51$ ) and linear pBA. At the same degrees of compression, LB films were transferred on the mica surface for AFM studies. Figure 7 shows two height images measured before and after compression of single brush molecules embedded in a matrix of linear pBA. Before compression, molecules demonstrate the characteristic rodlike conformation caused by steric repulsion of adsorbed side chains. Lateral compression induces desorption of the side chains and causes an instantaneous transition from the rodlike to globular conformation. ${ }^{12}$ The symmetric hemispherical shape of the gl obular conformation significantly differs from the tadpole conformation observed for the gradient brushes.

Three issues need to be discussed when comparing homogeneous and gradient brushes. First, grafting density of homogeneous brushes may be different compared to that of gradient brushes. Recently, we have shown that the multifunctional macroinitiators used for synthesis of cylindrical brushes with poly(methyl methacrylate) side chains have limited initiation efficiency and result in brushes with a grafting density of about $50 \% .{ }^{28}$ In a different study, homogeneous PBA brushes showed a higher density of $87 \%{ }^{29}$ Although these values are close to a grafting density of about $60 \%$ at the very end of the dense part of the studied gradient brushes (Figure 2), some uncertainty concerning the effect of the grafting density on the rod-globular transition still remains. To clarify this issue, we studied the compression behavior of two homogeneous brushes with a
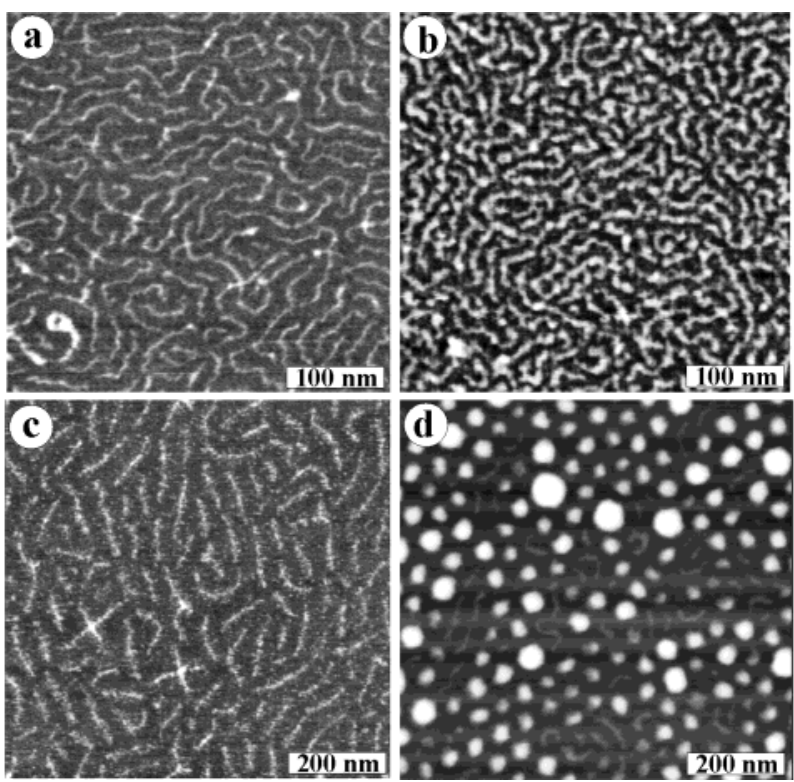

Figure 8. Conformational changes of homogeneous brushes with low grafting densities: $23 \%(a, b)$ and $65 \%(c, d)$. For the lower grafting density, molecules $(\mathrm{N}=710, \mathrm{n}=45)$ do not change their conformation. However, molecules with the higher grafting density $(\mathrm{N}=478, \mathrm{n}=75)$ demonstrate a transition from a rodlike to a globular conformation. Note the coexistence of both conformations in image $d$.

average grafting density of $23 \%$ and $65 \%$, respectively. Before compression, both types of molecules adopt a conformation of an extended semiflexible chain (Figure $8 \mathrm{a}, \mathrm{c})$. The mol ecules in Figure 8a with a lower grafting density of $23 \%$ are more flexible than the $65 \%$ grafted molecules in Figure 8c. After compression, the conformation of loose brush molecules did not change much, except the distance between molecules became smaller (Figure 8b). In contrast, the molecules with the higher grafting density demonstrated the characteristic rodgl obule transition (Figure 8d). Note that in this sample one can also see molecules that did not transform to globule. At this point we cannot provide a defined explanation for these species. It is tempting to assign them to brush molecules whose grafting density is I ower than the critical value below which the transition does not occur. If so, this would be another manifestation of the critical dependence of the transition on the molecular structure, which is controlled by the side-chain length and the grafting density. ${ }^{12}$ The coexistence of the rod and globular conformations is consistent with the tadpole conformation of the gradient brushes, where different grafting densities coexist in one molecule.

Second, tadpole conformation looks similar to some conformations observed in the transient region of the rod-globule phase transition of homogeneous brushes. ${ }^{12}$ In this region, globular conformation nucleates at different parts of rodlike molecules, though it preferably emerges at the backbone ends. Some of these transient conformations may have undulations at one of the ends; however, one should distinguish them from the tadpole conformation observed at equilibrium after the transition was completed.

Third, one might notice the difference in the side chain length of homogeneous brushes ( $n=51$ ) and gradient brushes $(n=74)$. It is difficult to synthesize different types of brush architectures with exactly the same side-chain length. However, the comparison of homogeneous and gradient brushes holds because the 
rod-gl obule transition is peculiar to pBA brushes with side chains longer than $\mathrm{n} \cong 20 .^{12}$ Moreover, both theory and experiment indicate that pBA brushes with longer side chains, e.g., $n=74$, would demonstrate the transition even more readily.

\section{Conclusions}

In conclusion, rod-globule transitions were observed only in the dense portion of individual gradient bottle brushes, which formed tadpole conformations under compression. Two conformations coexisting in one molecule are consistent with the first-order character of the transition. ${ }^{12}$ The obtained results confirm the role grafting density has on phase transitions of adsorbed bottle brushes. Dense molecular brushes undergo the transition more readily compare to loose brushes with lower grafting density. Moreover, distinct segregation of the globular and extended conformations within one molecule indicates critical dependence of the transition on the grafting density. Observing anisotropic behavior of gradient brushes demonstrates the ability to design brushes with inherent asymmetry and raises the possibility of controlling the conformation of brushes in such a way as to direct the motion of individual molecules.

Acknowledgment. This work was financially supported by the National Science Foundation ECS 0103307. The authors acknowledge the contribution of D. G. Shirvanyants, who developed software to analyze AFM images for conformation and size of molecules.

\section{References and Notes}

(1) Tsukahara, Y.; Tsutsumi, K.; Yamashita, Y.; Shimada, S. Macromol ecules 1990, 23, 5201-5208.

(2) Wintermantel, M.; Gerle, M.; Fischer, K.; Schmidt, M.; Wataoka, I.; Urakawa, H.; Kajiwara, K.; Tsukahara, Y. Macromol ecules 1996, 29, 978-983.

(3) Beers, K.; Gaynor, S. G.; Matyjaszewski, K.; Sheiko, S. S.; Möller, M. Macromolecules 1998, 31, 9413-9415.

(4) Börner, H. G.; Duran, D.; Matyjaszewski, K.; de Silva, M.; Sheiko, S. S. Macromolecules 2002, 35, 3389-3394.
(5) Schappacher, M.; Deffieux, A. Macromolecules 2000, 33, $7371-7377$

(6) Liu, Y.; Abetz, V.; Müller, A. H. E. Macromolecules 2003, 36, $7894-7898$.

(7) Birshtein, T. M.; Borisov. O. V.; Zhulina, E. B.; Khokhlov, A. R.; Yurasova, T. A. Polym. Sci. USSR 1987, 29, 1293-1300.

(8) Fredrickson, G. Macromol ecules 1993, 26, 2825-2831.

(9) Subbotin, A.; Saariaho, M.; Ikkala, O.; ten Brinke, G. Macromol. Rapid Commun. 2000, 21, 110-115.

(10) Gerle, M.; Fischer, K.; Schmidt, M.; Roos, S.; Müller, A. H. E.; Sheiko, S. S.; Prokhorova, S. A.; Möller, M. Macromolecules 1999, 32, 2629-2637.

(11) Sheiko, S. S.; Möller, M. Chem. Rev. 2001, 101, 4099-4123.

(12) Sheiko, S. S.; Prokhorova, S. A.; Beers, K. L.; Matyjaszewski, K.; Potemkin, I. I.; Khokhlov, A. R.; Möller, M. Macromolecules 2001, 34, 8354-8360.

(13) Porto, M.; Urbakh, M.; Klafter, J . Phys. Rev. Lett. 2000, 84, 6058-6061.

(14) J ülicher, F.; Armand, A.; Prost, J . Rev. Mod. Phys. 1997, 69 $1269-1281$

(15) Astumian, R. D. Science 1997, 276, 917-922.

(16) Howard, J. Nature (London) 1997, 389, 561-567.

(17) Matyjaszewski, K.; Ziegler, M. J .; Arehart, S. V.; Greszta, D.; Pakula, T. J . Phys. Org. Chem. 2000, 13, 775-786.

(18) Pakula, T.; Matyjaszewski, K. Macromol. Theory Simul. 1996, $5,987$.

(19) Madruga, E. L. Prog. Polym. Sci. 2002, 27, 1879-1924.

(20) Wang, J .-S.; Matyjaszewski, K. J . Am. Chem. Soc. 1995, 117, $5614-5615$

(21) Matyjaszewski, K.; Xia, J . Chem. Rev. 2001, 101, 2921-2990.

(22) Rivetti, C.; Guthold, M.; Bustamante, C. J . Mol. Biol. 1996, 264, 919-932.

(23) Hashimoto, T.; Okumura, A.; Tanabe, D. Macromolecules 2003, 36, 7324-7330.

(24) Prokhorova, S. A.; Sheiko, S. S.; Möller, M.; Ahn, C.-H.; Percec, V. Makromol. Rapid Commun. 1998, 19, 359-366.

(25) Maier, B.; Rädler, J . O. Phys. Rev. Lett. 1999, 82, 1911-1914.

(26) Camesano, T. A.; Wilkinson, K. J . Biomacromol ecules 2001, $2,1184-1191$

(27) Kiriy, A.; Gorodyska, G.; Minko, S.; J aeger, W.; Stepanek, P.; Stamm, M. J. Am. Chem. Soc. 2002, 124, 3218-3219.

(28) Neugebauer, D.; Matyjaszewski, K.; Goodhart, B.; Sheiko, S. S. How Dense are Cylindrical Brushes Grafted from a Multifunctional Macroinitiator? Submitted to Macromolecules.

(29) Sumerlin, B. S.; Neugebauer, D.; Matyjaszewski, K. I nitiation Efficiency in the Synthesis of Molecular Brushes by Grafting From via ATRP. Submitted to Macromolecules.

MA035989Z 